03,12

\title{
Электронный транспорт в нанопроволоках Те
}

\author{
() В.А. Березовец, Ю.А. Кумзеров, Ю.А. Фирсов \\ Физико-технический институт им. А.Ф. Иофрфе РАН, \\ Санкт-Петербург, Россия \\ E-mail: vaberez@inbox.ru
}

(Поступила в Редакцию 12 июля 2017 г.)

Проведено измерение температурной и магнетополевой зависимости вольтамперных характеристик образцов нанопроволок Те, изготовленных путем внедрения из расплава Те в поры хризотилового асбеста. Измерения проходили в широком интервале температур и магнитных полей. Анализ результатов таких измерений проводился путем сравнения их с предсказаниями теоретических моделей, разработанными для случая одномерных структур. Сделан вывод о том, что полученные зависимости наилучшим образом согласуются с предсказаниями теории о жидкости Латтинжера. Этот результат находится в соответствии с представлениями о том, что основной механизм протекания в таких одномерных нитях не зависит от материала, внедренного в поры, а зависит только от размерности проводящих нитей.

Работа частично поддержана программой Президиума РАН.

DOI: $10.21883 /$ FTT.2018.02.45376.221

\section{1. Введение}

В последние десятилетия возник большой интерес к исследованию систем с пониженной размерностью. Под этим термином следует понимать уменьшение геометрических размеров исследуемых объектов (в одном или нескольких направлениях) до величин, соизмеримых с длиной волны носителей тока в них. В таком случае перемещение носителей заряда в этих направлениях становится невозможным и размерность системы понижается. Данная работа посвящена исследованию проводимости одномерных (1D) нанопроволок теллура (Te), который впервые удалось внедрить в поры хризотилового асбеста. Хризотиловый асбест - изолятор, состоящий из тонких (диаметр $300 \AA$ ) нитей, в центре которых есть отверстия диаметром $50 \AA \AA$. Если внедрить в эту полость исследуемый полупроводник, то можно получить нанопроволоку этого материала. Для внедрения необходимо перевести полупроводник в расплавленное состояние и приложить внешнее давление [1]. Можно предположить, что электропроводность нанопроволок Те этого узкозонного естественного полупроводника будет обладать свойствами присущими жидкости Латтинжера, т.е. быть, в определенной мере, похожей на свойства нанопроволок из $\mathrm{InSb}$ [2]. Исследования проводились в широком интервале температур и магнитных полей.

Для описания проводимости в 1D-случае на данный момент нет точной модели, но есть точные решения для упрощенных моделей: модель Томонага [3], Латтинжеpa [4], латтинжерова жидкость (LL), о чем подробнее можно узнать в [5]. Общее для этих моделей состоит в следующем:

1. При учете слабого электрон-электронного $(e-e)$ взаимодействия пропадает ступенька в функции распределения Ферми при энергиях равных энергии Ферми $\left(\varepsilon_{\mathrm{F}}\right)$.
2. В области значений энергий $\varepsilon=\varepsilon_{\mathrm{F}}$ У функции плотности состояний $g(\varepsilon)$ образуется квазищель $\left(g\left(\varepsilon_{\mathrm{F}}\right)=0\right)$.

3. В области квазищели отсутствуют одночастичные возбуждения с зарядом $\pm e$ и спином $1 / 2$.

Несмотря на совпадение точных решений для этих моделей, тем не менее, предсказания поведения проводимости с изменением температуры и приложенного напряжения у них разные. В первой публикации [2] и последующих статьях [6,7], посвященных исследованию нанопроволок InSb в матрице хризотилового асбеста, авторы сделали вывод о том, что наиболее подходящей моделью для описания экспериментальных данных является модель LL.

\section{2. Техника эксперимента}

Для измерения вольт-амперных характеристик нитей Те в асбесте были приготовлены образцы в виде небольших пучков нитей (диаметр пучка $\sim 0.5 \mathrm{~mm}$ ), на торцевую часть которых термически напылялось золото. Напряжение смещения подавалось на образцы с аналогового интегратора, скорость изменения напряжения на выходе которого регулировалась компьютером. Для уменьшения влияния емкости проводов на величину измеряемых токов были использованы известные технические приемы. Ток через образец измерялся электрометром 642LN производство фирмы Keithley. Температура образцов регулировалась терморегулятором 340 Temperature Controller фирмы Lake Shore, сильное магнитное поле создавалось сверхпроводящим магнитом Oxford Instruments .

\section{3. Результаты измерений}

На рис. 1 представлены вольт-амперные характеристики $(\mathrm{BAX})$ одного из образцов наноразмерных нитей Те. 
Видно, что с понижением температуры от комнатной до температуры жидкого гелия вольт-амперные характеристики становятся нелинейными, а величины измеряемых значений тока падают на много порядков. На рис. 2 по-

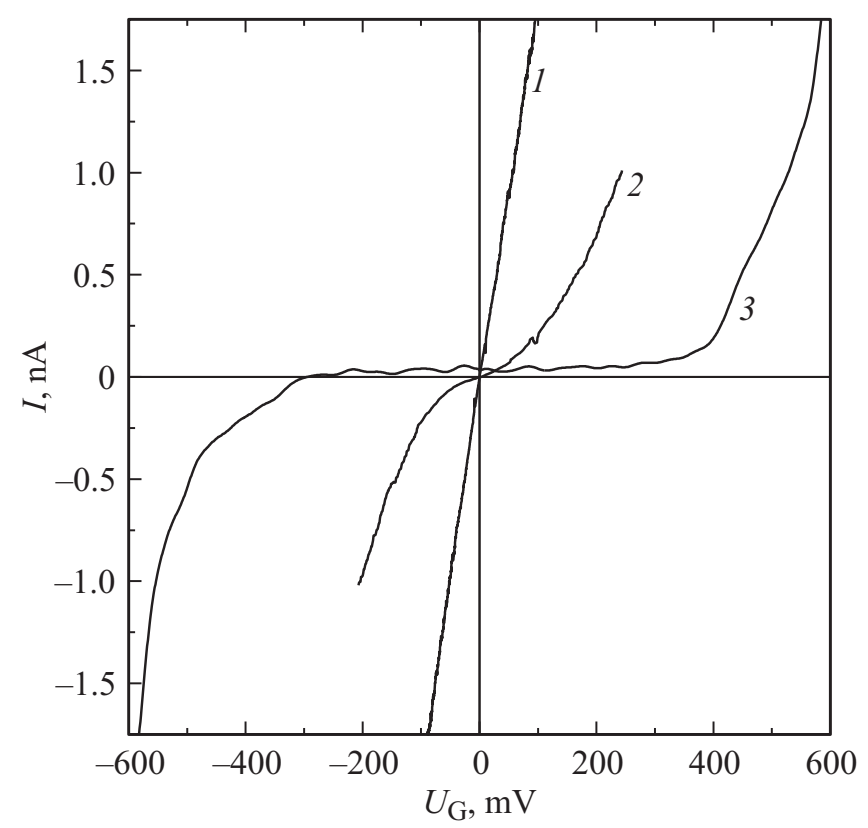

Рис. 1. ВAX образца Те в матрице асбеста при разных температурах. 1 соответствует $T=300 \mathrm{~K}$, характер зависимости - омический, расчетная величина сопротивления $6 \cdot 10^{6} \Omega$; зависимость 2 соответствует $T=77 \mathrm{~K}$, зависимость нелинейная, рисунок увеличен в $10^{3}$ раз; зависимость 3 соответствует $T=4.2 \mathrm{~K}$, зависимость нелинейная, рисунок увеличен в $10^{6}$ раз.

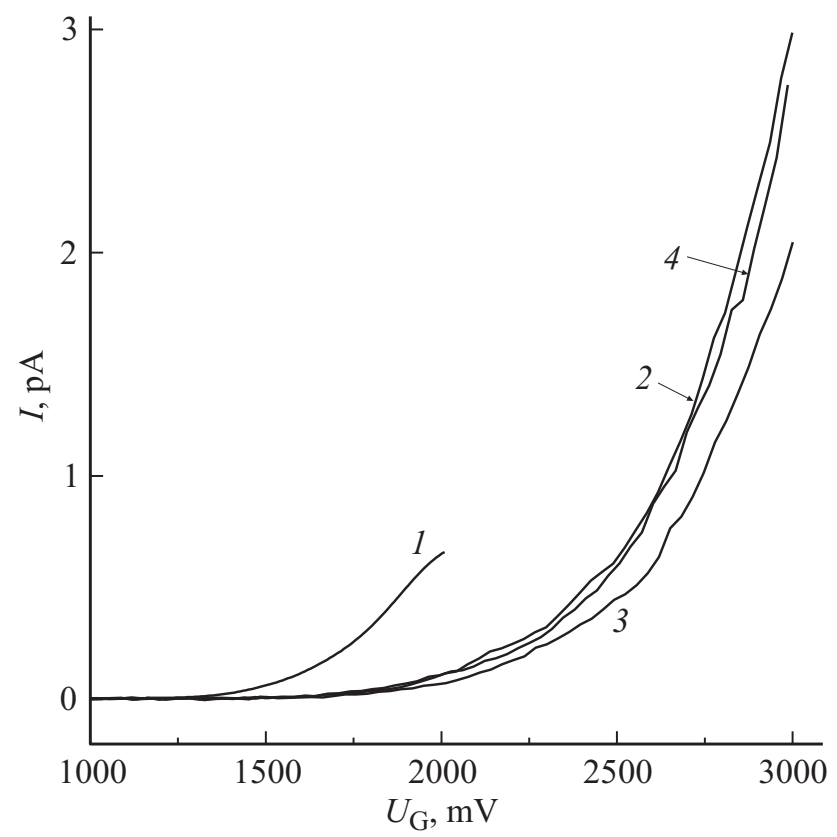

Рис. 2. Влияния внешнего магнитного поля на ВАХ одного из образцов: $1-T=4.2 \mathrm{~K}, B=0 ; 2-T=1.5 \mathrm{~K}, B=0$; $3-T=1.5 \mathrm{~K}, B=10 \mathrm{~T} ; 4-T=1.5 \mathrm{~K}$, после вывода магнитного поля. казана зависимость ВАХ, измеренная на другом образце при гелиевых температурах. Видно, что с понижением температуры от 4.2 до $1.5 \mathrm{~K}$ сопротивление образца существенно возросло, а включение магнитного поля индукцией $10 \mathrm{~T}$ (рис. 2,3) оказало действие аналогичное уменьшению температуры - сопротивление образца возросло по сравнению с исходным (без поля), т.е. наблюдается положительное магнетосопротивление. После вывода магнитного поля исходная зависимость ВАХ практически восстановилась (рис. 2, 2 и 4). Обнаруженное влияние поля на измеряемое сопротивление было подвержено подробному исследованию при разных ориентациях вектора индукции относительно оси асбестовых волокон. Оказалось, что при всех значениях напряжения смещения и независимо от взаимной ориентации вектора индукции магнитного поля и асбестовых волокон наблюдается уменьшение тока с ростом магнитного поля.

Эффект увеличения сопротивления полупроводниковых материалов с ростом магнитного поля хорошо известен для трехмерного случая. Изменение сопротивления под влиянием магнитного поля, вызывающего отклонение от прямолинейного движения носителей тока между актами рассеяния, происходит в таких магнитных полях, когда магнитная длина $L_{B} \propto \sqrt{\hbar / e B}$ для этих носителей меньше размеров образца в направлении перпендикулярном движению. В случае наноразмерных проволок Те таким размером является диаметр проволоки $(D \sim 5 \mathrm{~nm})$. Простые расчеты позволяют оценить магнитную длину $\mathrm{L}_{\mathrm{B}}$ в нашем случае: при $B=10 \mathrm{~T}$ эта величина составляет примерно $10 \mathrm{~nm}$, т. е. классическая модель влияния магнитного поля на электропроводность исследуемых образцов в этом случае неприменима, тем более, что увеличение сопротивления наблюдалось практически сразу с началом роста внешнего магнитного поля. В [6] в качестве возможного механизма влияния магнитного поля на сопротивление образцов рассматривается влияние магнитного поля на параметры, описывающие свойства LL. Чтобы выяснить насколько исследуемые нанопроволоки соответствуют этой модели LL, зависимости BAX нанопроволок от температуры были измерены в широком интервале температур.

Степенная зависимость проводимости $G \propto T^{\alpha}$ в случаe LL предсказывается для модели протекания туннельного тока между двумя каплями совершенной LL [7-9], для LL в длинной проволоке с атомами примеси [10] и в случае модели в виде 1D-вигнеровского кристалла [11]. Аналогично $I \propto V^{\beta}$ предсказывается для модели протекания туннельного тока между двумя каплями совершенной LL [7-9], для LL в длинной проволоке с атомами примеси [10] и в случае модели, учитывающей влияния кулоновской блокады [12-14]. В то же время экспоненциальная зависимость проводимости от температуры $G \propto \exp \left[v \cdot \ln \left(T_{0} / T\right)^{3 / 2}\right]$ была предсказана для эффекта туннелирования 1D заряженных частиц [15], для вигнеровких кристаллов $[16,17]$, и для чисто LL при наличии дальнодействующего кулоновского взаимодействия [15]. 


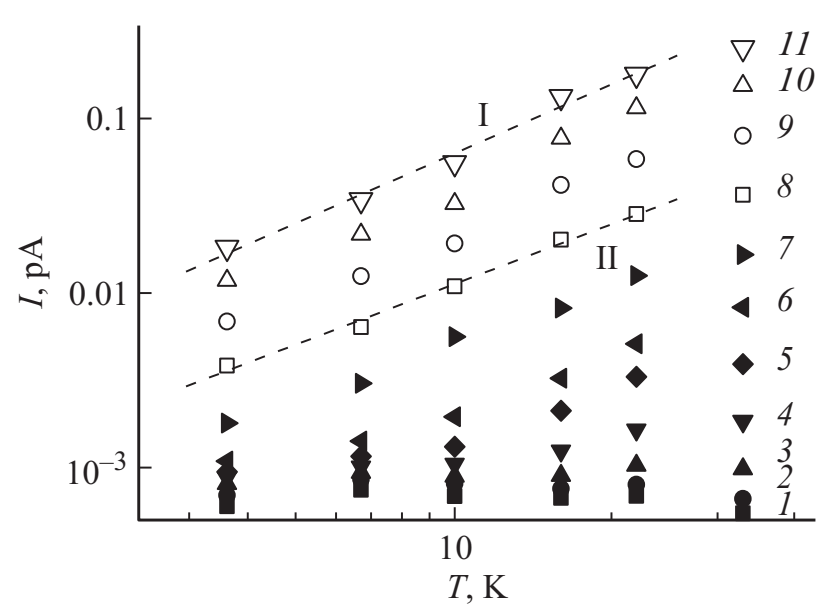

Рис. 3. Температурная зависимость тока через нанонити Те в матрице асбеста при разных напряжениях смещения (1-11 соответствуют напряжениям смещения: 0.5, 0.6, 0.7, $0.8,0.9,1,1.2,1.4,1.6,1.8,2.0 \mathrm{~V}$ соответственно). Для ВАХ, измеренных при двух напряжениях смещения $\left(U_{G}=1.4\right.$ и $\left.2 \mathrm{~V}\right)$, штриховыми линиями I и II показаны линейные аппроксимации результатов измерений.

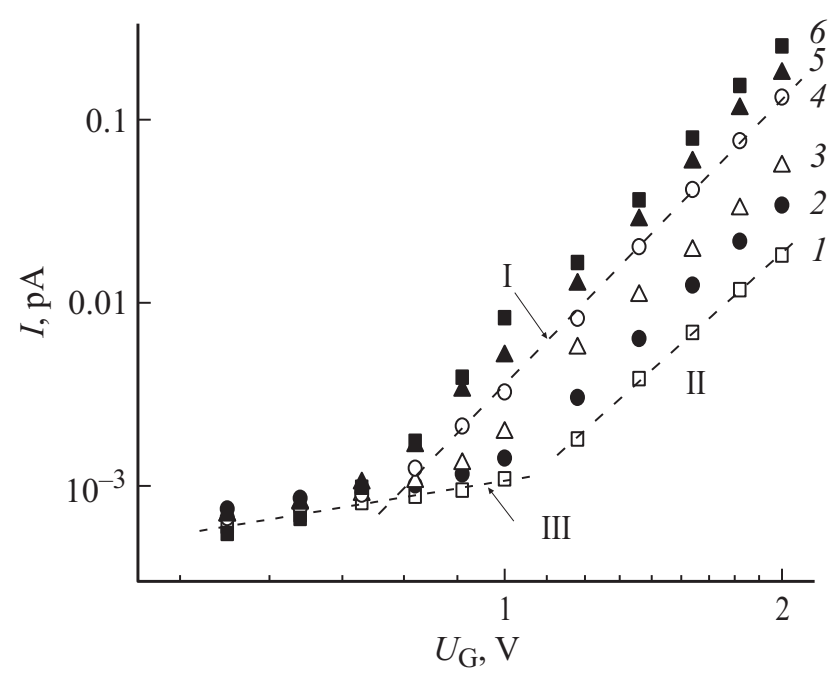

Рис. 4. BAX при разных температурах (номера $1-6$ соответствуют температурам $4.2,7,10,15,20,30 \mathrm{~K}$ соответственно). При $T=4.2 \mathrm{~K}$ нарастание тока происходит по степенному закону, но с двумя разными наклонами (II и III).

Мы предполагаем, что в нашем случае дальнодействующее кулоновское взаимодействие между электронами в каждой нанопроволоке может быть заэкранировано из-за кулоновского взаимодействия между электронами соседних проволок, что и приводит к короткодействующему межпроволочному $e-e$-взаимодействию, которое является главным условием в теории LL. Во всех остальных аспектах проволочки остаются независимы друг от друга. Ес-ли это предположение верно, то проводимость каждой проволочки будет определяться проводимостью системы последовательных LL капель, объединенных слабым кулоновским взаимодействием, а зависимость от температуры и напряжения смещения будет

$$
\begin{gathered}
G(T)=G_{T} N \frac{e^{2}}{\hbar}\left[\sum_{j=1}^{M}\left(\frac{\bar{\varepsilon}}{t_{j}}\right)^{2}\right]^{-1}\left(\frac{\kappa T}{\bar{\varepsilon}}\right)^{\alpha} \\
I(V)=C_{I} N \frac{e^{2}}{\hbar}\left(\frac{\bar{\varepsilon}}{c}\right)\left[\sum_{j=1}^{M}\left(\frac{\bar{\varepsilon}}{t_{j}}\right)^{\beta / 2}\right]^{-\beta}\left(\frac{e V}{\bar{\varepsilon}}\right)^{\beta},
\end{gathered}
$$

где $C_{T} C_{I} \sim 1, \tau_{j}$ описывает прозрачность $j$-ой слабой связи, $\bar{\varepsilon} \propto E_{\mathrm{F}}$, а $\alpha=2 / g-2, \beta=2 / g-1$, где $g-$ безразмерная константа.

Из приведенных выше выражений следует, что электропроводность от температуры должна зависеть степенным образом, как и электропроводность от напряжения смещения. На рис. 3 и 4 представлены результаты измерений перечисленных выше зависимостей, выполненных на одном из образцов. На рис. 3 показаны температурные зависимости тока от температуры при разных значениях напряжения смещения, а на рис. 4 штриховыми линиями показаны полевые зависимости тока через образец при разных значениях температуры. Для того чтобы исключить разогрев носителей тока в образцах максимальные величины тока в эксперименте не превышали $10^{-12} \mathrm{~A}$, а минимальные измеренные значения определялись уровнем шумов, который был ниже $5 \cdot 10^{-16} \mathrm{~A}$.

Видно, что, зависимости носят степенной характер, как и предсказывали формулы (1) и (2). На рис. 4 наблюдаются два наклона: один в более сильных электрических полях - линия II, которая соответствует поведению, предсказываемому формулой (2) и идентичному тому, что наблюдалось в [2]. В более слабых электрических полях зависимости оказались менее выраженными (линия III), а измеряемый ток был меньше 1 фемтоампера $\left(10^{-15} \mathrm{~A}\right)$. По нашему мнению, такая зависимость является следствием того, что при этих напряжениях еще не все нанопроволоки Те участвуют в процессе переноса заряда. Простые оценки дают число нитей в образце $\sim 10^{6}$, ток через них, если все включены и проводят, будет $1.6 \cdot 10^{-19} \mathrm{C} / \mathrm{s} \cdot 10^{6} \sim 10^{-13} \mathrm{~A}$, а учитывая, что не все нити из-за разрывов участвуют в проводимости, полученный результат вполне можно уменьшить на порядок. Тогда ток до $10^{-14} \mathrm{~A}$ будет нарастать с ростом напряжения из-за нарастания числа нитей, которые с увеличением напряжения окажутся включенными в процесс переноса электрического заряда.

\section{4. Заключение}

Результаты, представленные на рис. 3 и 4, доказывают, что исследуемые нити действительно по своим свойствам близки к модели LL, а поэтому и влияние магнитного поля на сопротивление нанопроволок Те может иметь ту же природу, что и в случае нанопроволок $\mathrm{InSb}$ [6]. 
Таким образом, из сравнения свойств нанопроволок $\mathrm{InSb}$ и Те можно утверждать, что макроскопические наборы параллельных нанопроволок, изготовленных из этих узкозонных полупроводников, имеют, в определенной мере, похожие физические свойства, соответствующие модели жидкости Латтинжера, для которой, вероятно, не столь важны свойства исходного материала нанопроволок, а важна, скорее, размерность системы, т. е. одномерность исследуемого объекта.

\section{Список литературы}

[1] M. Bokrath, D.H. Cobden, J. Lu, A.G. Rinzler, R.E. Smalley, L. Balents, P.L. Mc Euen. Nature 397, 598 (1999).

[2] S.V. Zaitsev-Zotov, Yu.A. Kumzerov, Yu.A. Firsov, P. Monceau. J. Phys: Condens. Matter 12, L303, (2000).

[3] S. Tomonaga. Prog. Theor. Phys. 5, 544, (1950).

[4] J.M. Luttinger. Phys. Rev. 119, 1153, (1960).

[5] Yu.A. Firsov, V.N. Prigodin, Chr. Seidel. Phys. Rep. 126, 5/6, 245, (1985).

[6] S.V. Zaitsev-Zotov, Yu.A. Kumzerov, Yu.A. Firsov, P. Monceau. Pisma Zh. Eksp. Teor. Fiz. 77, 3, 162 (2003); [JETF Lett. 77, 3, 135 (2003); condmat/0212633].

[7] C.L. Kane, M.P. Fisher. Phys. Rev. Lett. 68, 1220, (1992).

[8] C.L. Kane, M.P. Fisher. Phys. Rev. B 46, 15233, (1992).

[9] A. Furusaki, N. Nagaosa. Phys. Rev. B 47, 4631, (1993).

[10] S.R. Renn, D.A. Arovas. Phys. Rev. B 51, 16832, (1995).

[11] H. Maurey, T. Giamarchi. Phys. Rev. B 51, 10833, (1995).

[12] S.M. Girvin, L.I. Glazman, M. Jonson, D.R. Penn, M.D. Stiles, Phys. Rev. Lett. 64, 3183 (1990).

[13] K.A. Matveev, D. Yue, L.I. Glazman. Phys. Rev. Lett. 71, 3351, (1993).

[14] H. Maurey, T. Giamarchi. Europhys. Lett. 38, 681, (1997).

[15] N. Nagaosa, A. Furasaki. J. Phys. Soc. J. 63, 413, (1994).

[16] L.L. Glazman, I.M. Ruzin, B.I. Shklovskii. Phys. Rev. B 45, 8454, (1992).

[17] M. Fabrizio, A.O. Gegolin, S. Schedel. Phys. Rev. Lett. 72, 2235, (1994). 